\title{
Structure and stability of D-galactose/D-glucose-binding protein. The role of D-glucose binding and $\mathrm{Ca}$ ion depletion
}

\author{
Olga V. Stepanenko a,*, Olga I. Povarova ${ }^{a}$, Olesya V. Stepanenko ${ }^{\text {a }}$, Alexander V. Fonin ${ }^{\text {a }}$, \\ Irina M. Kuznetsova ${ }^{a}$, Konstantin K. Turoverov ${ }^{a}$, Maria Staiano ${ }^{b}$ and Sabato D'Auria ${ }^{b}$ \\ ${ }^{a}$ Institute of Cytology of the Russian Academy of Sciences, St. Petersburg, Russia \\ ${ }^{\mathrm{b}}$ Institute of Protein Biochemistry CNR, Napoli, Italy
}

\begin{abstract}
The effects of guanidine hydrochloride $(\mathrm{GdnHCl})$ on the structure and stability of the D-galactose/D-glucose-binding protein from Escherichia coli (GGBP) and its complex with D-glucose (GGBP/Glc) were investigated by intrinsic protein fluorescence and far-UV circular dichroism (CD). The role of calcium in the stability of the protein structure was also studied. It was shown that the processes of GGBP and GGBP/Glc unfolding induced by $\mathrm{GdnHCl}$ followed one-step reversible denaturation mechanism. The obtained data showed that the binding of glucose to GGBP resulted in an increase of the protein stability towards the actions of the $\mathrm{GdnHCl}$ which made protein unfolding more cooperative. The stabilities of GGBP alone, GGBP in the presence of glucose, GGBP-depleted calcium (GGBP-Ca), and GGBP/Glc-depleted calcium (GGBP/Glc-Ca) were characterized by difference of Gibbs free energies.
\end{abstract}

Keywords: D-galactose/D-glucose-binding protein, protein folding, protein stability, intrinsic fluorescence of proteins

\section{Introduction}

Protein folding remains one of the most intriguing problems of molecular biology [4,9]. When it seemed that we were quite near the explanation of protein folding in compact globular structure it appeared that many proteins in principle cannot have such structure alone, without their partners (other proteins, DNA molecules and inorganic ligand) $[13,14]$. In view of this new concept it is interesting to study the role of ligands in proteins structure and stability. The periplasmic binding proteins which can exist both in ligand-free and ligand-bound form seem to be an interesting candidate for such investigations.

This paper is focused on the study of the unfolding-refolding mechanisms of D-galactose/D-glucosebinding protein from Escherichia coli (GGBP) alone and in the presence of glucose (GGBP/Glc) induced by $\mathrm{GdnHCl}$. We also investigated the role of other ligand - calcium ion localized in the loop of the C-terminal domain, in the structure and folding process of protein. A significant protein conformational change as a result of ligand binding makes GGBP a good candidate as a sensitive element of biosensors

\footnotetext{
${ }^{*}$ Corresponding author: Dr. Olga V. Stepanenko, Institute of Cytology of the Russian Academy of Science, Tikhoretsky av. 4, 194064 St. Petersburg, Russia. Tel.: +7 81224719 57; Fax: +7 81224703 41; E-mail: sov@ mail.cytspb.rssi.ru.
} 
for glucose detection [3]. Thus investigation of GGBP structure and stability is of interest both for basic science and biotechnology as this protein is good candidate for a sensing element of the biosensor system for permanent non-invasive monitoring of glucose content in the blood of diabetic patients.

\section{Materials and methods}

\subsection{Materials}

GGBP from Escherichia coli was obtained and purified as described earlier [10]. The samples of D-glucose (Sigma, USA) and $\mathrm{GdnHCl}$ (Nacalai Tesque, Japan) were used without purification. GdnHCl concentration in solution was determined by Abbe refractometer (LOMO, Russia). The concentration of protein was $0.1-0.3 \mathrm{mg} / \mathrm{ml}$. D-glucose concentration was $10 \mathrm{mM}$ in all experiments with GGBP/Glc complex. For $\mathrm{Ca}^{2+}$ removal EDTA was added to its final concentration of $0.18 \mathrm{mM}$.

\subsection{Analysis of protein $3 D$ structure}

The comparison analysis of GGPB and GGBP/Glc complex was performed on the basis of PDB data [1]: files 2FW0.ent [2] and 2GBP.ent [15], respectively. The analysis of properties of microenvironment and localization of tryptophan and tyrosine residues in GGPB and GGBP/Glc was done as described earlier [12]. To evaluate the changes of the distance between each pair of atoms on ligand binding special program was elaborated.

\subsection{Fluorescence measurements}

Fluorescence experiments were carried out using a homemade spectrofluorimeters with steady-state and time-resolved excitation [11]. Fluorescence measurements were done in micro cells (101.016-QS $5 \times 5 \mathrm{~mm}$; Hellma, Germany). Fluorescence was excited at $297 \mathrm{~nm}$ and $280 \mathrm{~nm}$. The values of parameter $A=I_{320} / I_{365}$ characterizing the fluorescence spectra position $\left(I_{320}\right.$ and $I_{365}$ are fluorescence intensities at $\lambda_{\mathrm{em}}=320$ and $365 \mathrm{~nm}$, respectively [12]), and of fluorescence spectrum were corrected by the instrument sensitivity.

The equilibrium dependences of different fluorescent characteristics of GGBP on GdnHCl concentration were recorded after protein incubation in the solution of appropriate concentration at $4{ }^{\circ} \mathrm{C}$ overnight. For a more detailed analysis of the protein unfolding process and in order to determine the number of intermediate states appeared on the pathway from native to unfolded protein we used the method of phase diagrams [6].

\subsection{Circular dichroism measurements}

CD spectra were obtained with spectrophotometer Jasco-810 (Jasco, Japan). Far UV CD spectra were recorded in a $1 \mathrm{~mm}$ path length cell from 260 to $190 \mathrm{~nm}$. For all spectra, an average of 5 scans was obtained. The protein $\mathrm{CD}$ spectra were calculated taking into account the CD signal of the appropriate buffer solutions. 


\section{Results and discussion}

\subsection{Structure of GGBP and $G G B P / G l c$}

GGBP consists of two globular domains of practically identical topology connected by three mobile regions. Sugar-binding site is located in a deep cleft between two domains [15]. The central part of both domains consists of six $\beta$-sheets, surrounded by $\alpha$-helixes on both sides: two on one side and three on the other one. $\mathrm{Ca}^{2+}$ ion is localized in the loop of C-terminal domain (134-142 residues), forming coordination bonds with oxygen atoms of every second residue of this loop and with Glu 205 residue. Structure of Ca-binding center resembles "EF-hand" motive, typical for intracellular Ca-binding proteins $[2,15]$.

CD spectrum of GGBP shows two negative bands at 208 and $222 \mathrm{~nm}$, characteristic for proteins with high content of $\alpha$-helixes. The CD spectrum practically does not change upon D-glucose binding. Intrinsic fluorescence spectrum of GGBP is rather long-wave shifted $\left(\lambda_{\mathrm{em}}=345-346 \mathrm{~nm}\right.$ at $\lambda_{\mathrm{ex}}=$ $297 \mathrm{~nm}$ ). It consists with polar microenvironment of all five tryptophan residues of protein. Among these residues only one, Trp 183, is oriented to the inner part of the cleft which serves as a ligand binding site. This tryptophan residue and Phe 16 residue form the sugar binding aromatic pocket. Sugar molecule incorporates between the indole and benzene rings of Trp 183 and Phe 16. Small, 1-2 nm, blue-shift of GGBP fluorescence spectra on ligand binding can be explained by the change of microenvironment of this tryptophan residue. Changes in the microenvironments of other tryptophan residues are negligible. Really, though the relative position of domains changes significantly upon the binding of D-glucose, their structures remain practically unchanged. The maximal variation in the distances between $\mathrm{C} \alpha$ atoms on complex formation was observed for residues Ser 46 and Asp 190, Ala 194 and Ser 197 (13.1, 13.1, $13.0 \AA$ ). If the atoms of side chains are included in observation then the maximum change of the distance at complex formation is found for NZ Lys 47 and O atom of Asp 190 (19.7 $)$. These data are of interest for development of biosensor system for continuous non-invasive detection of glucose level in the blood of diabetic patients with GGBP as sensing element, since they provide information about part of the protein matrix that should be labeled with extrinsic fluorescence probes to obtain large spectral changes upon glucose binding.

\subsection{GGBP, GGBP/Glc and their forms with depleted $\mathrm{Ca}^{2+}$ folding-unfolding induced by $\mathrm{GdnHCl}$}

The denaturing action of $\mathrm{GdnHCl}$ on the structure of GGBP, GGBP/Glc and their forms with depleted $\mathrm{Ca}^{2+}$ (GGBP-Ca and GGBP/Glc-Ca, respectively) was studied by protein tryptophan intrinsic fluorescence and by $\mathrm{CD}$ in far UV-region. To characterize the rate of GGBP unfolding induced by $\mathrm{GdnHCl}$ the kinetic changes of the intensity of intrinsic fluorescence $\left(I_{350}\right)$ of GGBP and GGBP/Glc were recorded at different concentrations of GdnHCl. It was obtained that the process of GGBP/Glc unfolding was much slower than that of GGBP. Experimental data on the unfolding kinetics of GGBP-Ca and GGBP/Glc-Ca induced by $\mathrm{GdnHCl}$ showed that GGBP-Ca structure is less stable with respect to GGBP. At the same time the presence of glucose (GGBP/Glc-Ca) increases the protein stability against the denaturing action of $\mathrm{GdnHCl}$.

The comparison of the transition curves measured by different structural probes (such as fluorescence intensity, parameter $A$, anisotropy and ellipticity at $222 \mathrm{~nm}$ ) showed that GdnHCl-induced unfolding of both GGBP and GGBP/Glc is a one-stage unfolding process (Fig. 1). This is also proved by the linear character of the parametric dependences between fluorescence intensity recorded at 320 and $365 \mathrm{~nm}$. 


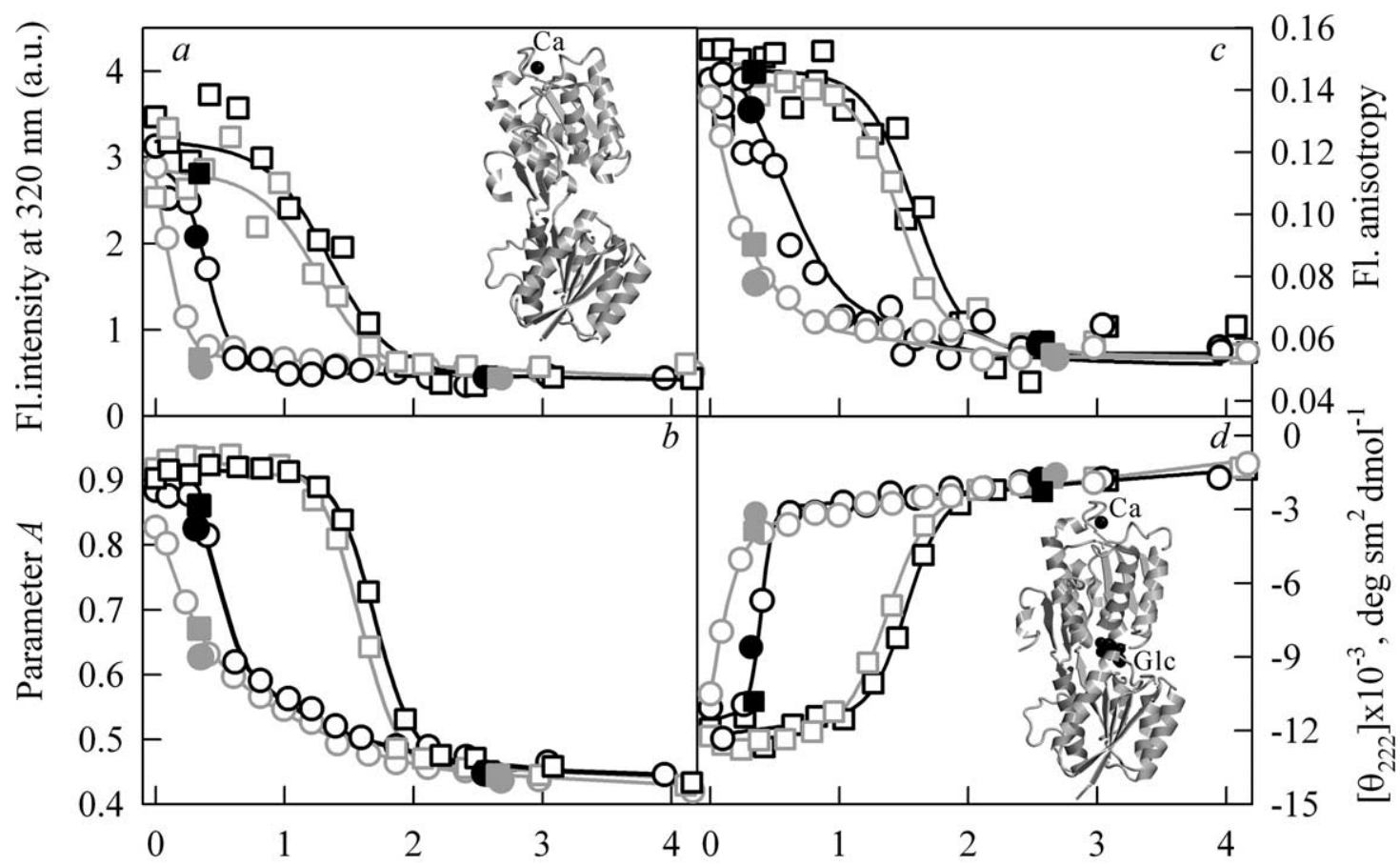

$\mathrm{GdnHCl}, \mathrm{M}$

$\mathrm{GdnHCl}, \mathrm{M}$

Fig. 1. Conformational changes of GGBP and GGBP-Ca (black and grey circles, respectively) and GGBP/Glc and GGBP/Glc-Ca (black and grey squares, respectively) induced by GdnHCl. (a) Changes in fluorescence intensity recorded at $320 \mathrm{~nm}$; (b) Changes of parameter $A$. (c) Changes of fluorescence anisotropy. Excitation wavelength was $297 \mathrm{~nm}$. (d) Changes in ellipticity at $222 \mathrm{~nm}$. Open symbols - unfolding, closed - refolding. Inserts to panels (a) and (d): 3D structure of GGBP and its complex with D-glucose GGBP/Glc, respectively. Ca ion and molecule of bounded D-glucose are presented as van der Vaalse spheres. The figure is done on the basis of PDB [1] data: files 2GBP.ent [15] and 2FWO.ent [2] using graphical software VMD [5], Raster 3D [7].

The mid-point of the unfolding of GGBP/Glc (1.34 M of GdnHCl) is shifted to larger values of $\mathrm{GdnHCl}$ concentrations as compared to GGBP alone $(0.36 \mathrm{M}$ of $\mathrm{GdnHCl} ;$ Fig. 1$)$. These results certify stabilizing action of glucose on the protein structure.

The equilibrium dependences of all fluorescent characteristics and far UV CD obtained on the pathway of GGBP renaturation from the completely unfolded state in the presence of $3 \mathrm{M} \mathrm{GdnHCl}$ coincide well with the corresponding denaturation curves (Fig. 1). This suggests the reversibility of the GGBP unfolding induced by GdnHCl. Furthermore, even at $0.5 \mathrm{M}$ of GdnHCl GGBP recovers the ability to bind glucose. It is also proved by the increase of fluorescence intensity to the level of fluorescence of native complex of GGBP with the ligand if renaturation is performed in the presence of D-glucose.

The equilibrium dependences of fluorescence intensity of GGBP and GGBP/Glc complex as well as its forms with depleted $\mathrm{Ca}^{2+}$ upon $\mathrm{GdnHCl}$ additions were used for the evaluation of the protein free energy differences in native and unfolded states $\Delta G_{\mathrm{N}-\mathrm{U}}(0)$. The calculation was performed according to the standard scheme [8]. The obtained data reveal that $\mathrm{Ca}^{2+}$ has a significant stabilizing effect on the protein structure. The difference of free energy of GGBP-Ca cannot be defined accurately due to complexity of estimation of the fluorescence intensity of protein native state (Fig. 1). The $\Delta G_{\mathrm{N}-\mathrm{U}}(0)$ value of GGBP-Ca should be considered being close to zero. The glucose binding results in an essential 
stabilization of GGBP structure both with $\mathrm{Ca}^{2+}$ ion and without it. (The $\Delta G_{\mathrm{N}-\mathrm{U}}(0)$ values of GGBP, GGBP/Glc and GGBP/Glc-Ca are equal to 8.0, 14.2 and $14.2 \mathrm{~kJ} / \mathrm{mol}$, respectively.)

\section{Acknowledgements}

This work was supported by NATO Grant CLG.983088 (MS and IMK), Contracts with FASI (02.512.11.2277 and 02.740.11.5141) and FAE (P1198), MCB RAS and "Leading Scientific School of RF" (1961.2008.4), Grants from SPb administration MKN-185 (OVS, 2009) and NTD-3 (OVS, 2009).

\section{References}

[1] H.M. Berman, K. Henrick and H. Nakamura, Announcing the worldwide protein data bank, Nature Struct. Biol. 10 (2003), 980.

[2] M.J. Borrok, L.L. Kiessling and K.T. Forest, Conformational changes of d-glucose/D-galactose binding protein illuminated by apo and ultrahigh resolution ligand-bound structures, Prot. Sci. 16 (2007), 1032-1041.

[3] S. D'Auria and J.R. Lakowicz, Enzyme fluorescence as a sensing tool: new perspectives in biotechnology, Curr. Opin. Biotechnol. 12 (2001), 99-104.

[4] C.M. Dobson, Protein folding and misfolding, Nature 426 (2003), 884-890.

[5] W. Humphrey, A. Dalke and K. Schulten, VMD: visual molecular dynamics, J. Mol. Graphics 14 (1996), 33-38.

[6] I.M. Kuznetsova, K.K. Turoverov and V.N. Uversky, Use of the phase diagram method to analyze the protein unfoldingrefolding reactions: fishing out the "invisible" intermediates, J. Proteome Res. 3 (2004), 485-494.

[7] E.A. Merritt and D.J. Bacon, Raster3D: photorealistic molecular graphics, Methods Enzymol. 277 (1977), 505-524.

[8] B. Nolting, Protein Folding Kinetics. Biophysical Methods, Springer-Verlag, Berlin, 1999, p. 191.

[9] O.B. Ptitsyn, Molten globule and protein folding, Adv. Protein Chem. 47 (1995), 83-229.

[10] L. Tolosa, I. Gryczynski, L.R. Eichhorn, J.D. Dattelbaum, F.N. Castellano, G. Rao and J.R. Lakowicz, Glucose sensor for low-cost lifetime-based sensing using a genetically engineered protein, Anal. Biochem. 267 (1999), 114-120.

[11] K.K. Turoverov, A.G. Biktashev, A.V. Dorofeiuk and I.M. Kuznetsova, A complex of apparatus and programs for the measurement of spectral, polarization and kinetic characteristics of fluorescence in solution, Tsitologiia 40 (1998), 806817.

[12] K.K. Turoverov and I.M. Kuznetsova, Intrinsic fluorescence of actin, J. Fluorescence 13 (2003), 41-57.

[13] K.K. Turoverov, I.M. Kuznetsova and V.N. Uversky, The protein kingdom extended: native globular and native partially or completely disordered proteins, their folding, supramolecular complex formation, and aggregation, Prog. Biophys. Mol. Biol. (2010), in press; doi: 10.1016/j.pbiomolbio.2010.01.003.

[14] V.N. Uversky, C.J. Oldfield and A.K. Dunker, Intrinsically disordered proteins in human diseases: introducing the D2 concept, Annu. Rev. Biophys. 37 (2008), 215-246.

[15] N.K. Vyas, M.N. Vyas and F.A. Quiocho, Sugar and signal-transducer binding sites of the Escherichia coli galactose chemoreceptor protein, Science 242 (1988), 1290-1295. 


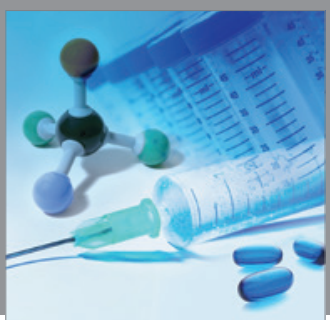

International Journal of

Medicinal Chemistry

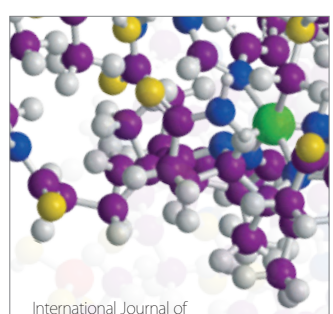

Carbohydrate Chemistry

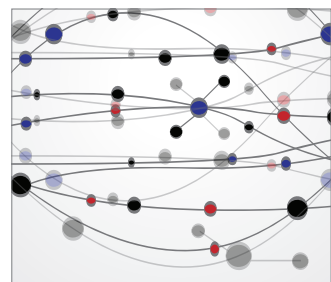

The Scientific World Journal
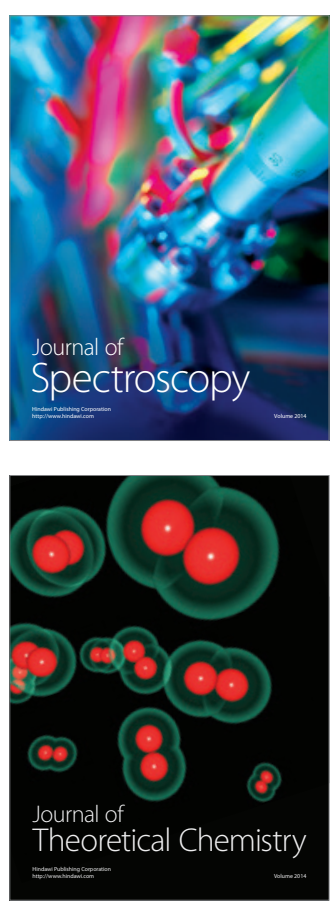
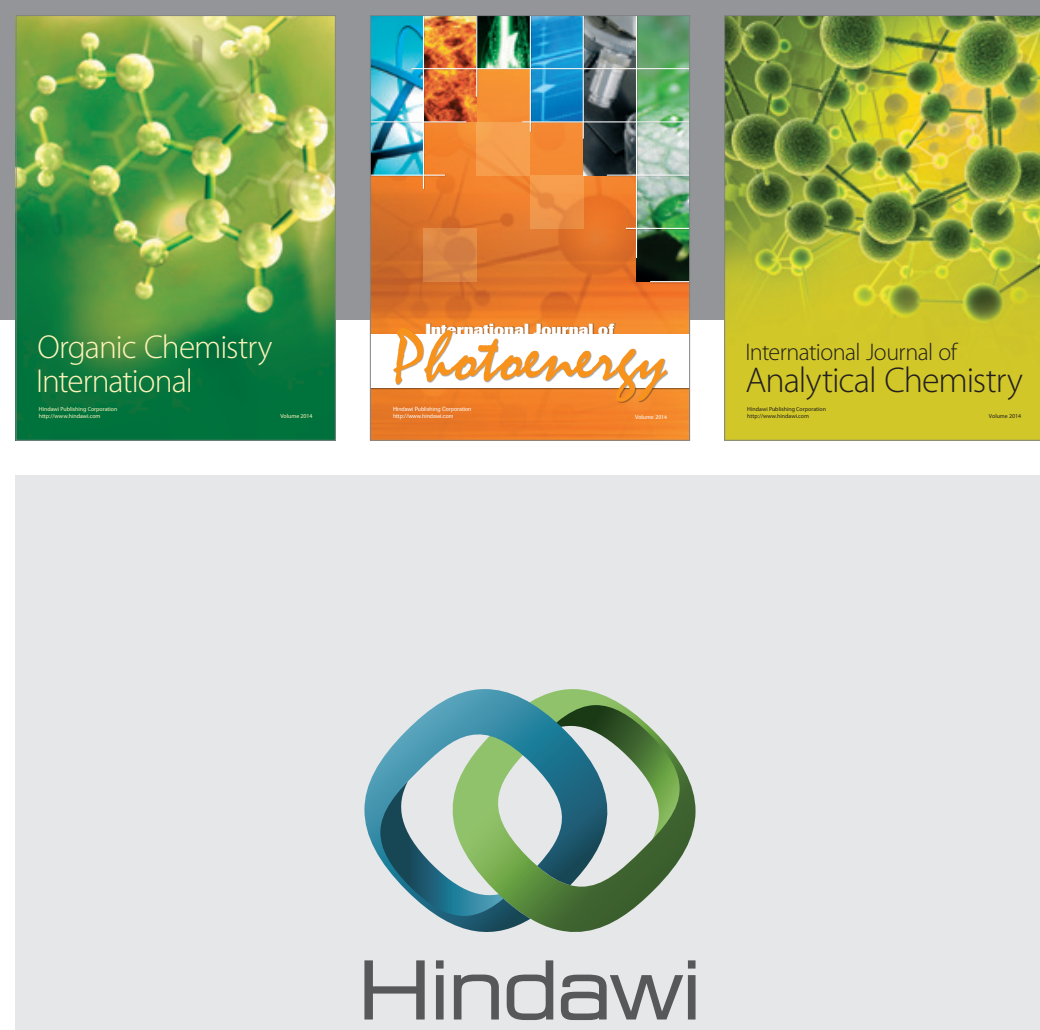

Submit your manuscripts at

http://www.hindawi.com
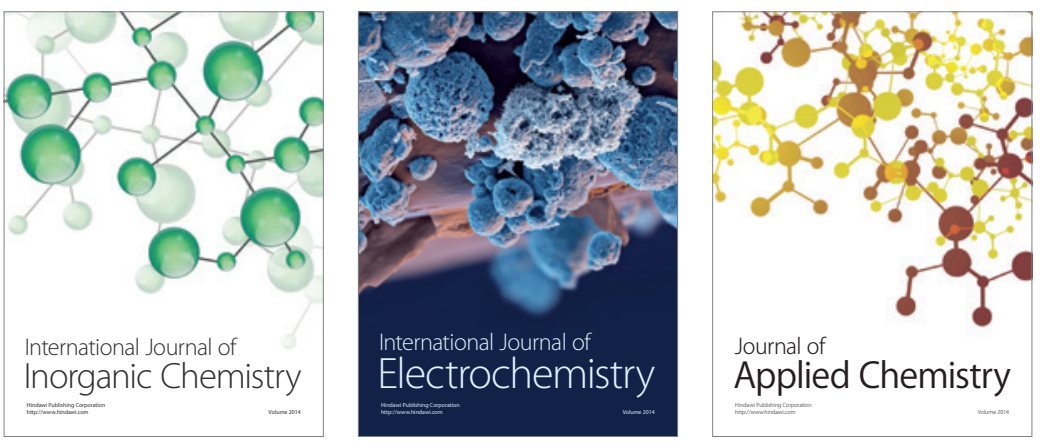

Journal of

Applied Chemistry
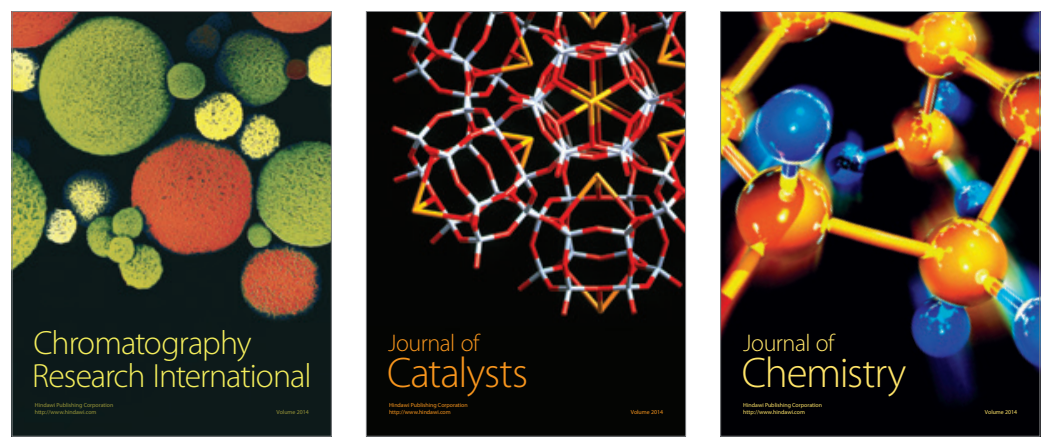
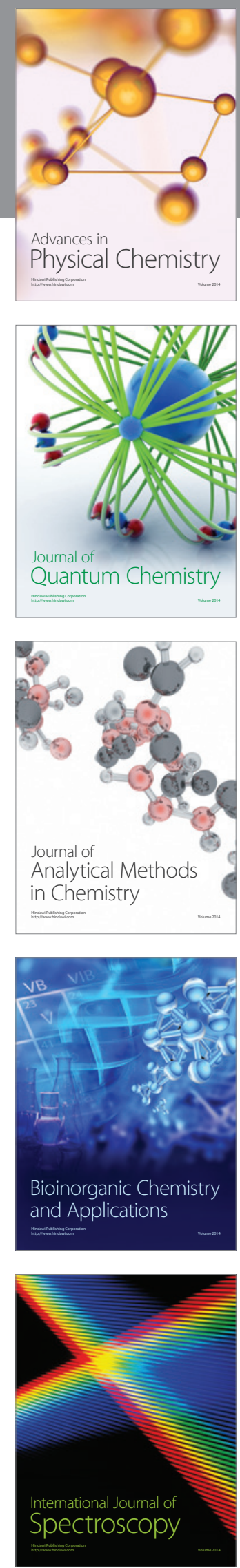